\title{
Correlation between histone acetylation and expression of Notch1 in human lung carcinoma and its possible role in combined small-cell lung carcinoma
}

\author{
Wael Abdo Hassan 1,2, Shin-ichiro Takebayashi ${ }^{3}$, Mohamed Osama Ali Abdalla ${ }^{4,5}$, Kosuke Fujino ${ }^{1,6}$, Shinji Kudoh, \\ Yamoto Motooka ${ }^{1,6}$, Yonosuke Sato ${ }^{1}$, Yoshiki Naito ${ }^{7}$, Koichi Higaki ${ }^{8}$, Joeji Wakimoto ${ }^{9}$, Seiji Okada ${ }^{10}$, Mituyoshi Nakao ${ }^{4}$, \\ Yuichi Ishikawa ${ }^{11}$ and Takaaki Ito'
}

Combined small-cell lung carcinoma (CSCLC) is composed of small-cell lung carcinoma (SCLC) admixed with non-small-cell lung carcinoma (NSCLC). Evaluating the molecular differences between SCLC and NSCLC could lead to a better understanding of the pathogenesis of such neoplasms. Therefore, in this study, we investigated the correlation between histone acetylation and Notch1 expression in lung carcinoma. Using chromatin immunoprecipitation (ChIP) assay, we measured the level of acetylated histone $\mathrm{H} 3$ around the promoter region of Notch1 in SCLC and NSCLC cells. We then treated SCLC cells with trichostatin A (TSA) and characterized the level of histone H3 acetylation at Notch1. In addition, TSA-treated cells were injected into immune-compromised mice, for analysis of the ex vivo tumor xenograft phenotype. The level of acetylated histone H3 surrounding the Notch1 promoter was lower in lung cancer cells not expressing Notch1. Tumors originated from TSA-treated SCLC cells occasionally formed an epithelial-like glandular arrangement of cells; with Notch 1 expression and decreased expression of neuroendocrine (NE) markers. Histone deacetylation around the promoter region of Notch1 inhibits Notch1 protein expression in SCLC and the restoration of Notch1 expression in SCLC leads to the concurrent appearance of epithelial-like areas within the SCLC, which could provide a possible mechanism for histogenesis of CSCLC.

Laboratory Investigation (2017) 97, 913-921; doi:10.1038/labinvest.2017.36; published online 17 April 2017

Combined small-cell lung carcinoma (CSCLC) is defined by the World Health Organization (WHO) as small-cell carcinoma (SCLC) combined with any of the non-small-cell lung carcinoma (NSCLC) types including adenocarcinoma (ADC), squamous cell carcinoma (SCC), and large-cell carcinoma (LCC). ${ }^{1}$ SCLC (including the cSCLC variant) comprises $15-20 \%$ of all lung cancers, with cSCLC accounting for $4-6 \%$, with a high incidence of mortality. ${ }^{2}$ Combined tumors in any organ raise biologic questions about its origin, pathogenesis, and treatment. In addition, cSCLC is considered as a type of SCLC, possibly due to the neuroendocrine (NE) differentiation of both tumor components, ${ }^{3}$ yet the difference in their morphology needs further clarification. Due to the lack of cSCLC cell lines, studying such a unique type of lung carcinoma in vitro requires comparison of cellular models of both SCLC and NSCLC, to mimic the clinical scenario.

One of the most significant molecular differences between SCLC and NSCLC is the activation of Notch1 signaling in the latter. ${ }^{4-6}$ Notch1 has different roles in SCLC and NSCLC, as we previously showed, ${ }^{6,7}$ and its induction induced 'epithelial-like' features in SCLC tissue sections. ${ }^{6}$ The exact mechanism of Notch1 silencing in SCLC remains unclear, although $25 \%$ of SCLC cases have mutations in Notch-related genes. ${ }^{8}$ Moreover, histone acetylation has been reported to

\footnotetext{
'Department of Pathology and Experimental Medicine, Graduate School of Medical Sciences, Kumamoto University, Kumamoto, Japan; ${ }^{2}$ Department of Pathology, Faculty of Medicine, Suez Canal University, Ismailia, Egypt; ${ }^{3}$ Department of Biochemistry and Proteomics, Graduate School of Medicine, Mie University, Mie, Japan; ${ }^{4}$ Department of Medical Cell Biology, Institute of Molecular Embryology and Genetics, Kumamoto University, Japan; ${ }^{5}$ Department of Clinical Pathology, Faculty of Medicine, Suez Canal University, Ismailia, Egypt; ${ }^{6}$ Department of Thoracic Surgery, Graduate School of Medical Sciences, Kumamoto University, Kumamoto, Japan; ${ }^{7}$ Department of Pathology, Kurume University School of Medicine, Kurume, Japan; ${ }^{8}$ Division of Pathology, St Mary Hospital, Kurume, Japan; ${ }^{9}$ Division of Pathology, Minami Kyushu National Hospital, Kagoshima, Japan; ${ }^{10}$ Department of Hematopoiesis, Center for AIDS Research, Kumamoto University, Kumamoto, Japan and ${ }^{11}$ Division of Pathology, The Cancer Institute of Japanese Foundation for Cancer Research, Tokyo, Japan

Correspondence: Professor T Ito, MD, DMSc, Department of Pathology and Experimental Medicine, Kumamoto University, Graduate School of Medical Sciences, Honjo 1-1-1, Chuo-ku, Kumamoto 860-8556, Japan.

E-mail: takaito@kumamoto-u.ac.jp

Received 4 November 2016; revised 27 February 2017; accepted 9 March 2017
} 
affect the development and progression of lung carcinoma in both SCLC and NSCLC. ${ }^{9-12}$ In addition, restoration of Notch1 protein expression in SCLC cells, after treatment with a histone deacetylase inhibitor (HDACi), ${ }^{13-15}$ suggests that histone deacetylation is responsible for epigenetic inactivation of Notch1 in SCLC. As HDACi treatment induces global DNA histone acetylation, ${ }^{16}$ comparison of the level of histone acetylation at the promoter region of Notch1 in Notch1-expressing and non-Notch1-expressing cells is necessary to confirm the role of histone acetylation in Notch1 expression. In the present study, we examined the expression of Notch1 in SCLC and NSCLC cell lines and tissue sections, as well as in surgically resected cSCLC tissue sections. In addition, to clarify the contribution of histone deacetylation to the silencing of Notch1 in SCLC, we examined the level of acetylated histone $\mathrm{H} 3$ surrounding the promoter region of Notch1 in SCLC compared to that of NSCLC cell lines, by quantitative analysis using a chromatin immunoprecipitation (ChIP) assay. Moreover, we used an HDACi; trichostatin A (TSA), to treat SCLC cells, and then examined the level of acetylated histone $\mathrm{H} 3$ at the promoter region of Notch1, to clarify whether or not HDACi specifically induces acetylation at the Notch1 promoter region. In addition, we xenotransplanted TSA-treated SCLC cells into immune-compromised mice, and examined the change in the morphology of tumor cells after restoration of Notch1 expression. Moreover, we examined the effect of TSA treatment on some of SCLC biological properties including cell adhesion, epithelial mesenchymal transition, and cell proliferation. Our findings reveal the role of epigenetic modulation of Notch1 expression in SCLC and NSCLC, which may lead to a better understanding of the origin of cSCLC, and thus, therapeutic targeting for such lung neoplasms.

\section{MATERIALS AND METHODS Cell Lines}

The following human lung cancer cell lines were purchased from the American Type Cell Collection (Manassas, VA): H69, H69AR, H889, and HI668 (SCLC), H358 and H1975 (adenocarcinoma; ADC), and $\mathrm{H} 226$ and $\mathrm{H} 2170$ (squamous cell carcinoma; SCC). H15 SCC and SBC-3 SCLC cell lines were a generous gift from Dr Makato Suzuki (Department of Respiratory Surgery, Graduate School of Medical Sciences, Kumamoto University). The A549 ADC cell line was provided by RIKEN Bio Resource Center (Tsukuba, Japan). All growth media were purchased from Wako Pure Chemical Industries (Osaka, Japan) and supplemented with $1 \%$ penicillin/ streptomycin (Sigma Aldrich, Ontario, Canada). A549 cells were grown in DMEM supplemented with 10\% FBS (Hyclone, Logan, UT). SBC-3 cells were grown in EMEM with 10\% FBS. H69AR cells were grown in RPMI 1640 medium supplemented with $2 \mathrm{mM} \mathrm{L}$-glutamine, $10 \mathrm{nM}$ HEPES, $1 \mathrm{mM}$ sodium pyruvate, $4.5 \mathrm{~g} / \mathrm{l}$ glucose, $1.5 \mathrm{~g} / \mathrm{l}$ sodium bicarbonate, and 20\% FBS. The rest of the cells were grown in similar RPIMI 1640 medium, but were supplemented with $10 \%$ FBS. All cells were incubated at $37^{\circ} \mathrm{C}$ in $5 \% \mathrm{CO}_{2}$ and saturated humidity. Supplementary Table S1 summarizes the type of cells used.

\section{Western Blotting Analysis}

Cells were prepared for western blot (WB) as previously described. ${ }^{17}$ A list of primary antibodies used is listed in Supplementary Table S2. The membrane was then washed and incubated with the respective secondary antibodies conjugated with peroxidase (Amersham Pharmacia Biotech, Buckinghamshire, UK) for $1 \mathrm{~h}$, and the immune complex was visualized with an ECL system (Santa Cruz Biotechnology, Santa Cruz, CA).

\section{ChIP Assay}

The promoter region of the Notch 1 gene was determined from the location of the transcriptional start site and the genomic structure surrounding this site. ${ }^{18}$ In brief, two sets of PCR primers were designed to cover the promoter region. In addition, a PCR primer for another non-promoter region on the Notch1 gene was designed and served as a negative control. A list of primers used is listed in Supplementary Table S3. ChIP assays were performed using anti-acetylated histone H3 (Millipore, Darmstadt, Germany) and normal rabbit IgG (sc2027, Santa Cruz Biotechnology), as previously described. ${ }^{19}$ Two million cells were harvested, fixed in $1 \%$ formaldehyde, and sonicated to produce chromatin fragments 300 to $500 \mathrm{bp}$ in length. The precipitated DNAs were extracted by phenol-chloroform, and then purified. Quantitative PCR (qPCR) reactions included the following: $5 \mu \mathrm{l}$ of ChIP product, $10 \mu \mathrm{l}$ of 2X SYBR green PCR master mix (Applied Biosystem, Carlsbad, CA, USA, 4309155), and $25 \mathrm{nM}$ of each primer. The concentration of the ChIP samples was calculated as a percent of the input DNA isolated from immunoprecipitated chromatin, and whole cell extracts (input) were used as templates for PCR. Then, the fold enrichment of each sample was calculated as the difference between each sample and the negative control sample. In cells treated with TSA, levels of relative expression $>2.0$ were considered to be a significant increase in Notch1 expression from TSA treatment, as previously described. ${ }^{20}$ Individual assays were repeated three times to confirm the reproducibility of the experiment.

\section{HDACi Treatment}

For cell proliferation and in vitro enzyme inhibition studies, a $2 \mathrm{mM}$ solution of Trichostatin A (TSA) (Sigma Aldrich) in DMSO was prepared and stored at $-20^{\circ} \mathrm{C}$ until use. Cells were grown to $50 \%$ confluency and then treated with TSA with various concentrations $(100,300$, and $1000 \mathrm{nM})$ for $24 \mathrm{~h}$. Another set of cells was treated with DMSO as a vehicle control. The proper concentration of TSA was adjusted so that cell growth was inhibited to $30 \%$ confluency. ${ }^{21,22}$

\section{Immunofluorescence Microscopy}

Cells were plated in 24-well plates and treated as previously described. ${ }^{17} \mathrm{~A}$ list of primary antibodies used is listed in 
Supplementary Table S2. Cells were incubated with the appropriate secondary antibodies (Alexa Flour, Molecular Probes, Eugene, OR) and examined using a fluorescent microscope (Olympus, Tokyo, Japan).

\section{Reverse Transcription-PCR}

Total RNA was isolated from cells using Trizol reagent (Invitrogen, Carlsbad, CA), in accordance to the manufacturer's instructions, and then processed as previously described. ${ }^{6,16}$ A list of primers used and conditions of RT-PCR is listed in Supplementary Table S4. Amplified DNA fragments were separated on a $1.5 \%$ agarose gel and identified after ethidium bromide staining.

\section{Tumor Xenograft Growth In vivo and Histopathological Evaluation}

$\operatorname{Rag} 2(-/-)$ :Jak3 $(-/-)$ mice at 5 to 6 weeks of age, were used for all studies involving animals. All animal work was done according to Institutional Animal Care and Use Committee guidelines. The viability of H1688 cells was determined by trypan blue, and only cell populations with $90 \%$ or greater viability were used for this investigation. Two groups of mice were injected subcutaneously in the back; one group was injected with a $2 \times 10^{6}$ TSA-treated cell population, and the other group was injected with an equal number of a DMSOtreated cell population, for a period of 28 days. Individual tumors were split for fixation in $4 \%$ paraformaldehyde and then used for histology and immunostaining. Representative data were obtained from six mice per experimental group.

In addition, tissue samples of lung $\operatorname{ADC}(n=10)$, SCC $(n=5)$, SCLC $(n=10)$, and cSCLC $(n=7)$ were obtained from anonymous cases of lung cancer patients, who were surgically treated at Kumamoto University and Minami Kyushu National Hospitals, following the guidelines of the Ethics Committee of Kumamoto University (approval number 342). All samples were fixed with $10 \%$ formalin and embedded in paraffin. Tissue sets were stained with hematoxylin and eosin (H\&E), Alcian blue, and immunohistochemical (IHC) staining, as previously described. ${ }^{6}$ A list of primary antibodies used is listed in Supplementary Table S2. Appropriate positive control and negative control tissue slides were subsequently performed. All histopathological and IHC stained slides were reviewed by the researcher and another independent pathologist in a blinded fashion. The immunoreaction for Notch1 was assessed in cell membranes and/or cytoplasm, while that for NE markers (synaptophysin (SYP), chromogranin A (CGA), and neuron-specific enolase (NSE)) was evaluated in cell cytoplasm. A modified semi-quantitative method was used to assess the staining intensity, as previously described. ${ }^{3}$

\section{Statistical Analysis}

The results were expressed as the means \pm the standard error of triplicate determinations. The differences in the mean values between the groups were analyzed by two-tailed statistical analysis using Student's $t$-tests. $P$-values less than 0.05 were considered to be statistically significant. Statistical analysis was performed using the JMP9 software program (SAS Institute, Cary, NC, USA).

\section{RESULTS}

\section{Expression of Notch1 in Lung Cancer}

Notch1 and its activated intracellular domain (NICD) were often detected by WB analysis of NSCLC cells. In contrast, all SCLC cells, except H69AR and SBC-3, lacked Notch1 expression (Figure 1a). To confirm our in vitro findings, IHC staining of human lung carcinoma tissue was performed. SCLC cells showed negative Notch1 expression $(n=10 / 10)$, while NSCLC cells showed positive Notch1 expression; with strong staining in the membranes of ADC cells $(n=10 / 10)$, and weak staining in the cytosol of SCC cells $(n=9 / 9)$ (Figure $1 \mathrm{~b}$ and Table 1). Moreover, NICD showed similar IHC expression patterns (Supplementary Figure 1A). Regarding CSCLC, four cases were found to be SCLC with ADC, and three cases were found to be SCLC with SCC. The expression patterns of Notch1 in cSCLC cases was different in either component of CSCLC, and similar to that of pure SCLC and NSCLC cases, with expression of Notch1 negative in the SCLC component; while strongly positive in the ADC component and weakly positive in the SCC component (Figure 1c and Table 1).

\section{Expression of Acetylated Histone $\mathrm{H} 3$ and the Level of its Expression Around the Notch1 Promoter in SCLC and NSCLC Cells}

We detected the level of expression of acetylated histone H3 in lung cancer cells by WB. There was a significant difference in the expression of acetylated histone H3 between SCLC and NSCLC cells, with the greatest expression detected in the former. In addition, within SCLC cells, we observed lower expression of acetylated histone $\mathrm{H} 3$ in SBC-3 cells (Notch1 protein-expressing cells) compared to other non-Notch1 protein-expressing cells (H69, H889, and H1688), which may signify a relationship between histone acetylation and the expression of Notch1 in SCLC cells (Figure 2a). Next, using ChIP assay, the level of acetylation of histone $\mathrm{H} 3$ surrounding the promoter region of Notch1 was examined in three groups of lung cancer cells: SCLC with no Notch1 expression; H69 and H1688, SCLC with Notch1 expression; H69AR and SBC-3, and NSCLC; A549 (ADC) and H2170 (SCC). We found that the expression of acetylated histone $\mathrm{H} 3$ was reduced in $\mathrm{H} 69$ and $\mathrm{H} 1688$ cells, increased by about 3-fold in H69AR and SBC-3 cells, increased by about 20-fold in A549 cells, and increased by 10 -fold in $\mathrm{H} 2170$ cells (Table 2 and Figure 2b). These observations indicate that histone $\mathrm{H} 3$ acetylation is associated with Notch1 protein expression in lung cancer cell lines. 


\section{TSA Effect on Notch1 Expression}

To further clarify the role of histone acetylation in controlling Notch1 expression in SCLC cells, we treated H69 and H1688 cells with TSA concentrations of $300 \mathrm{nM}$ and $1000 \mathrm{nM}$, respectively, for $24 \mathrm{~h}$, which resulted in a 30-50\% inhibition of cell growth in vitro. The expression of acetylated histones $\mathrm{H} 3$ and $\mathrm{H} 4$ detected by WB and immunofluorescence (IF) confirmed the successful histone acetylation. We found

a

\begin{tabular}{|lllllllllll|}
\hline & \multicolumn{3}{|c|}{ SCLC cell lines } & \multicolumn{3}{c|}{ ADC cell lines } & \multicolumn{3}{c|}{ SCC cell lines } \\
\hline H69 & H69AR & H889 & H1688 & SBC-3 & A549 & H358 & H1975 & H226 & H2170 & H15
\end{tabular}

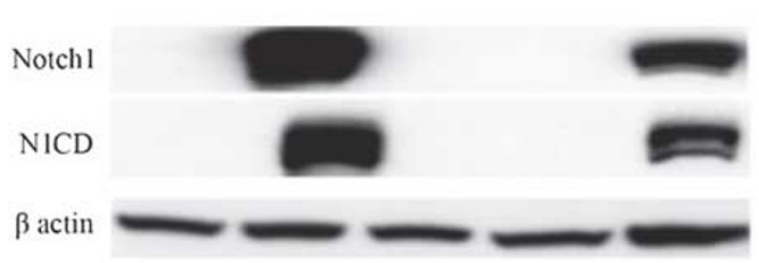

b

SCLC
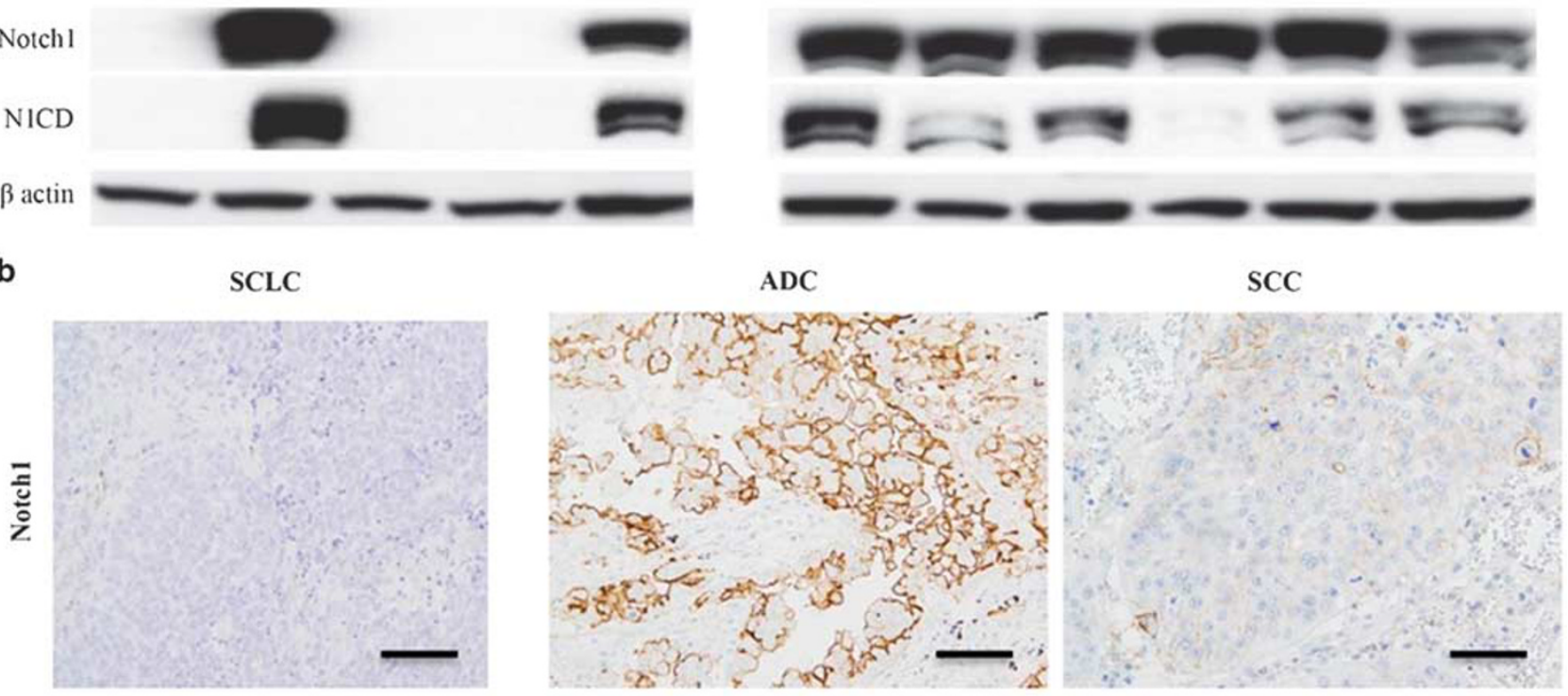

c
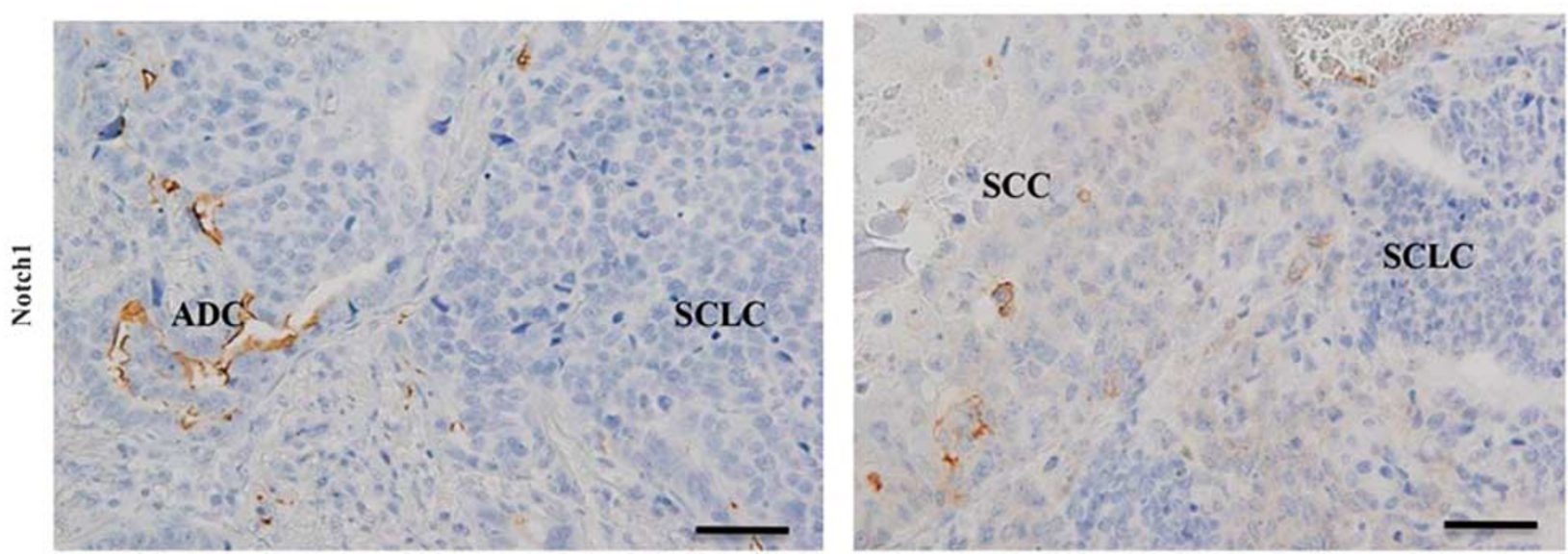

Figure 1 Expression of Notch1 in lung cancer cell lines and tissue sections. (a) Western blot (WB) analysis of Notch1 and its intracellular domain (NICD) in human small-cell lung cancer (SCLC) and non-small-cell lung cancer (NSCLC) cell lines, adenocarcinoma (ADC), and squamous cell carcinoma (SCC). Cells cultured in the same conditions were harvested and supplied for WB. Notch1 and NICD were mainly detected in NSCLC cells, and only in H69AR and SBC-3 SCLC cells. The expression of $\beta$-actin was used as an internal control. The experiment was performed in triplicate. (b) Representative photos for immunohistochemical (IHC) staining of Notch1 in SCLC and NSCLC tissue sections. The immunoreaction (brown) for Notch1 was detected in the membrane and cytosol. SCLC cells showed negative Notch1 expression. In NSCLC, immunoreactivity of for Notch1 was strongly positive in the cell membrane of ADC, and weakly positive in SCC cytosol. Original magnification: $\times 100$ (scale bar $100 \mu \mathrm{m}$ ). SCLC, small-cell lung carcinoma; ADC, adenocarcinoma, SCC; squamous cell carcinoma. (c) Representative photos for IHC staining for Notch1 in combined SCLC (cSCLC) tissue sections. The first photo represents a case of CSCLC with an ADC as the NSCLC component, while the second one represents another case of cSCLC with a SCC as the NSCLC component. Note the differences between the two components of CSCLC; Notch1 was expressed in the NSCLC component (more in ADC than in SCC) and was absent in the SCLC component. Original magnification: $\times 100$ (scale bar $100 \mu \mathrm{m}$ ). ADC, adenocarcinoma; SCC, squamous cell carcinoma; SCLC, small-cell lung carcinoma. 
increased expression of Notch1 by WB, IF, and RT-PCR in treated cells (Figure 3a).

\section{Acetylation Level of Histone H3 Around the Notch1 Promoter in TSA-Treated Cells}

After confirming the ability of TSA to induce Notch1 protein expression in SCLC, we tested whether such effect is caused by TSA direct acetylation of the promoter region of Notch1. We compared the level of histone $\mathrm{H} 3$ acetylation at the Notch1 promoter in untreated SCLC cells and in cells treated with TSA, by ChIP assay using antibodies against acetylated histone H3. In H1688 cells with induction of Notch1 expression by TSA treatment, the level of histone H3 acetylation at the Notch1 promoter was increased $(>2.0$, underlined in Table 3 and Supplementary Table S5). In contrast, H69 cells with Notch1 induction after TSA treatment did not show a significant change in $\mathrm{H} 3$ acetylation at the Notch1 promoter. These observations indicate that TSA treatment may lead to an increase in the level of $\mathrm{H3}$ acetylation at the promoter region of Notch1, leading to Notch1 protein expression.

\section{Morphology of Tumors Arising From Xenotransplantation of H1688 Cells Treated with TSA}

We examined whether Notch1 induction in SCLC after TSA treatment changes the morphology of tumor cells, leading to the development of an NSCLC component within the tumor. We have previously shown that induction of Notch1 by plasmid transfection in SCLC cells induced an 'epithelial-like' cell arrangement. ${ }^{6}$ After confirming sustained expression of Notch1 in H1688 cells treated with TSA for 28 days by WB analysis (data not shown), we xenotransplanted a $2 \times 10^{6}$ TSA-treated cell population into immunocompromised mice, as described in the materials and methods, and examined the tumor morphology after 28 days. The subcutaneous tumors, originating from TSA-treated cells, showed multiple foci of glandular arrangement of cells, and Alcian blue staining at their luminal surfaces (Figure 3b). In addition, TSA-treated cells showed positive Notch1 staining (Figure 3c) and decreased expression of NE markers SYP (Figure 3c), NSE, and CGA. These findings indicate the possible acquirement of epithelial-like features, in cells that regained Notch1 expression after TSA treatment.

\section{Effect of TSA Treatment on SCLC Biological Properties}

Treatment of SCLC cell with TSA resulted in an increased expression of $\mathrm{E}$ cadherin (E cad), and a decreased expression of epithelial mesenchymal transition markers slug and Vimentin (Vim), as shown by WB analysis and IF (Supplementary Figure 1B). In addition, in TSA-treated H69 cells, the expression of snail was decreased. Moreover, cells treated with TSA showed decreased expression of phosphorylated histone $\mathrm{H} 3$ (p-H3), a cell proliferation marker, and increased expression of cleaved caspase 3 (CCsp3), a cell apoptotic marker. 
a

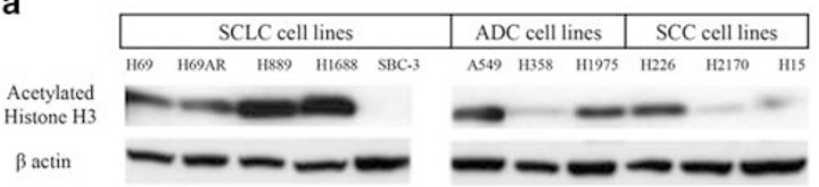

b

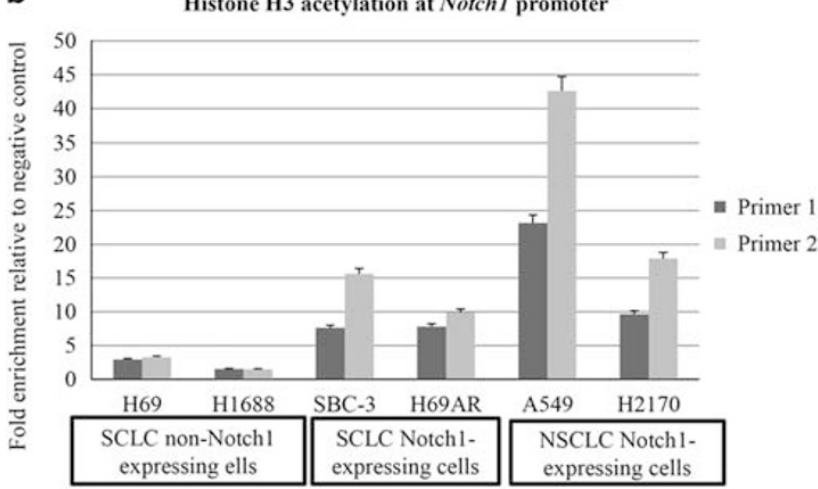

Figure 2 Expression of acetylated histone $\mathrm{H} 3$ and the correlation between Notch1 expression and acetylated histone $\mathrm{H} 3$ in lung cancer cell lines. (a) WB analysis for the expression of acetylated histone $\mathrm{H} 3$ in lung cancer cells. Acetylated histone $\mathrm{H} 3$ was more often expressed in SCLC than in NSCLC cells. Note the lower expression of acetylated histones in SBC-3 cells compared to other SCLC cells. The expression of $\beta$-actin was used as an internal control. The experiment was performed in triplicate. (b) Enrichment of the promoter region of the Notch1 gene with acetylated histone $\mathrm{H} 3$ in Notch1 expressing cells. After cell fixation and harvesting, chromatin DNA was isolated, sheared by sonication, and immune-precipitated with monoclonal antibody against acetylated histone $\mathrm{H} 3$. The resulting enriched genomic DNA was purified and used in ChIP-qPCR. Two sets of PCR primers were designed to cover the promoter region of Notch1. In addition, a PCR primer for another non-promoter region on the Notch1 gene was designed and served as a negative control. The results are expressed as the fold enrichment of each sample from the negative control sample, and plotted on the $X$ axis. Note the increase in the level of acetylated histone $\mathrm{H} 3$ in cells expressing the Notch 1 protein, both in SCLC cells (H69AR and SBC-3) and NSCLC cells (A549 and H2170), when compared to SCLC cells not expressing the Notch1 protein (H69 and H1688). In addition, A549 ADC cells showed the highest level of acetylated histone $\mathrm{H} 3$, which correlates with the strong Notch1 protein expression in the membrane of ADC tissue sections (Figure 1b).

\section{DISCUSSION}

Lung cancer is the most frequent and one of the deadliest cancer types, with $>1.1$ million deaths annually worldwide. ${ }^{1}$ Among its various types, SCLC accounts for $\sim 15 \%$ of cases, and regardless of its stage, the current prognosis for patients with SCLC is unsatisfactory, despite improvements in diagnosis and therapy. ${ }^{23}$ The new 2015 WHO classification of lung tumors ${ }^{1}$ listed cSCLC as a variant of SCLC, consisting predominantly of SCLC with some areas of NSCLC. The presence of NSCLC components is usually seen in up to $30 \%$ of SCLC case autopsies, whereas SCLC components are seen in approximately $14 \%$ of NSCLC tumors with EGFR mutations. ${ }^{24}$ In addition, cSCLC occurs after treatment of 'pure' SCLC with chemotherapy and/or radiation. ${ }^{25}$ The
Table 2 Histone H3 Acetylation at the promoter region of the Notch1 gene in human lung cancer cell lines

\begin{tabular}{lrr}
\hline $\begin{array}{l}\text { Cell } \\
\text { line }\end{array}$ & \multicolumn{2}{c}{ Acetylated histone H3 } \\
\cline { 2 - 3 } & \multicolumn{1}{c}{ Primer 1 } & Primer 2 \\
\hline H69 & $2.92 \pm 1.45$ & $3.28 \pm 0.45$ \\
H1688 & $1.53 \pm 0.18$ & $1.47 \pm 0.37$ \\
H69AR & $7.63 \pm 0.76$ & $15.63 \pm 2.98$ \\
SBC-3 & $7.82 \pm 0.27$ & $9.91 \pm 1.29$ \\
A549 & $23.51 \pm 2.80$ & $42.91 \pm 4.49$ \\
H2170 & $9.67 \pm 3.39$ & $17.88 \pm 4.26$ \\
\hline
\end{tabular}

The fold enrichment of the histone acetylation level. The average from three independent experiments \pm s.d.

present study represents a trial to better understand the mechanisms by which both SCLC and NSCLC components could evolve in the same tumor.

Due to the lack of cSCLC cell lines, we evaluated cellular models of SCLC and NSCLC (Supplementary Table S4), as well as tissue sections from cSCLC cases. Besides the morphological, genetic, and biological discrepancies between SCLC and NSCLC, we were interested in the difference between their expression of Notch $1,{ }^{4-6,26}$ as Notch signaling is one of the main regulator systems for SCLC carcinogenesis and differentiation, ${ }^{8}$ and whether an epigenetic effect, especially histone acetylation, could influence Notch1 expression in lung carcinoma cells, based on the observation that treating SCLC cells with HDACi results in restoration of Notch1 expression. ${ }^{13-15}$ Moreover, we investigated whether SCLC cells that regained Notch1 expression show features of NSCLC, as we previously showed that xenotransplanted SCLC cells transfected with Notch1 NICD plasmid showed glandular-like cell arrangement, with decreased NE markers and positive Alcian blue staining. ${ }^{6}$

Among the cells used, H69 is a drug-sensitive SCLC cell line, which when treated with increasing concentrations of doxorubicin, the surviving cells, named H69AR, develop a multidrug-resistant phenotype. ${ }^{27}$ We have previously shown that H69AR cells weakly express neuroendocrine markers, and strongly express MRP1, E cadherin, and BCL-2 proteins, compared to H69 cells, which could play a role in the multidrug resistance of H69AR cells. ${ }^{6,7}$ The nature of development of such drug resistance is multifactorial, involving gene mutations and epigenetic alterations, resulting in DNA methylation and altered miRNA. ${ }^{28}$ Elevated levels of histone acetylation in the MRP1 promoter in SCLC drugresistant cells has previously been described. ${ }^{29}$ In addition, overexpression of miR-134, miR-379, and miR-495 in H69AR cells lead to re-sensitization of cells to cisplatin, etoposide, and doxorubicin. ${ }^{30}$ 
a

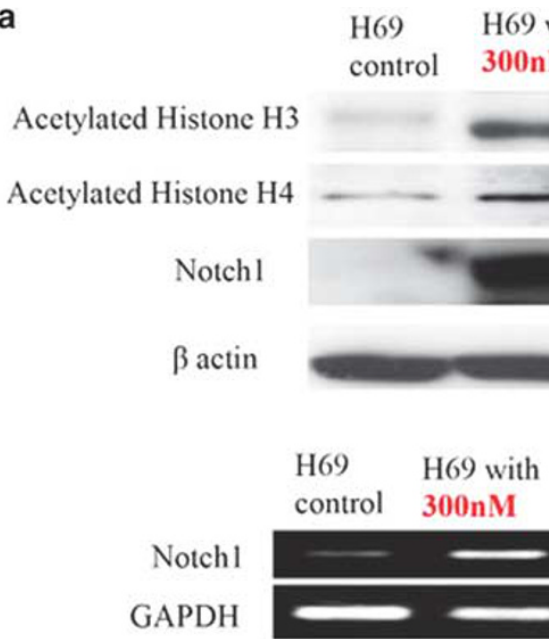

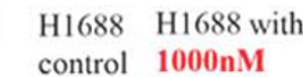

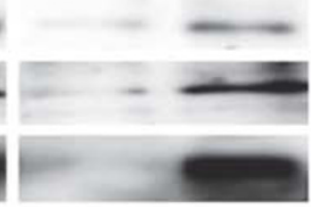

.
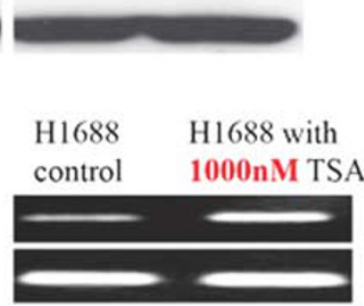

b
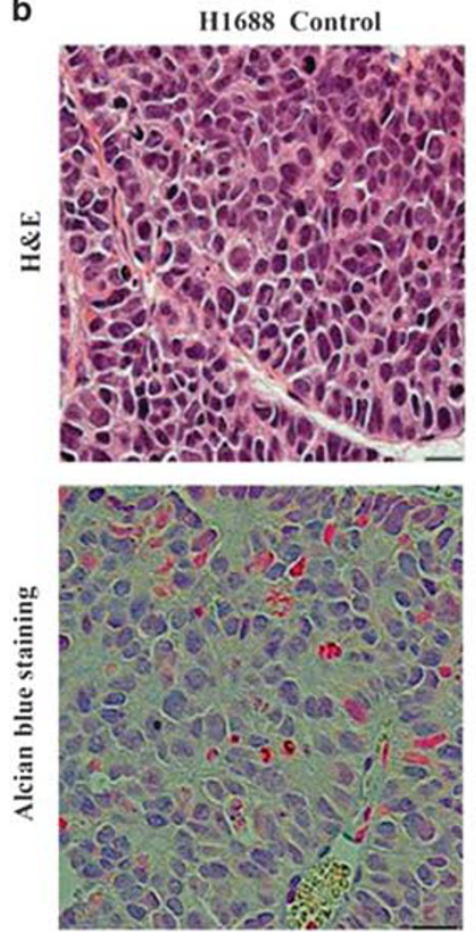
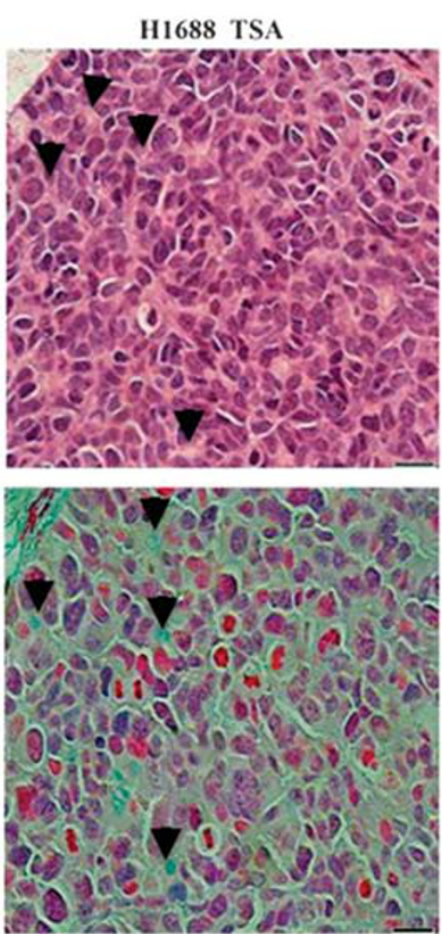

C

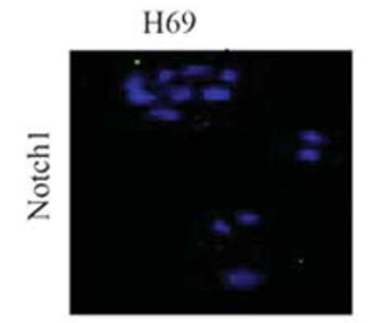

H69 with $300 \mathrm{nM}$ TSA
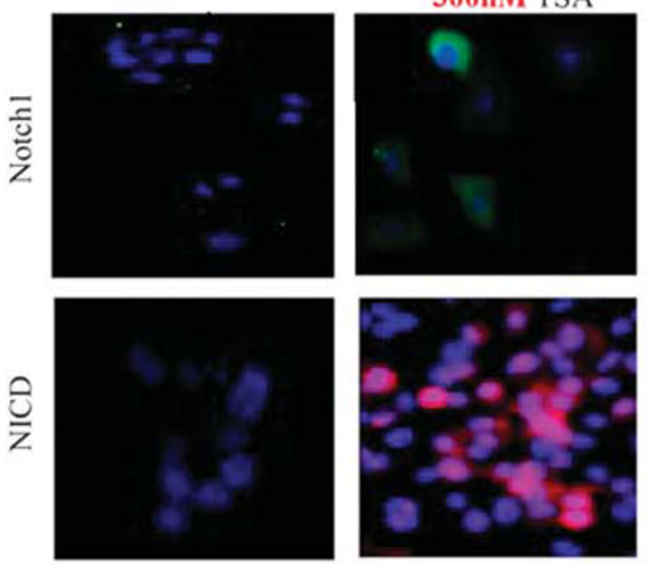
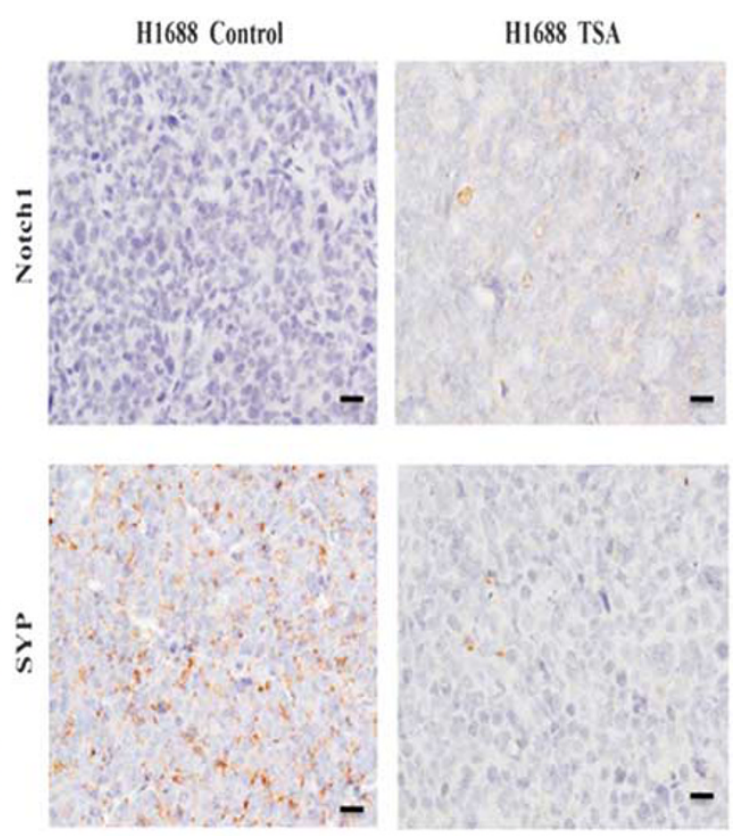

Figure 3 The effect of trichostatin A (TSA) on Notch1 expression and the morphology of SCLC cells. (a) The effect of TSA on Notch1 expression in H69 and H1688 SCLC cells was evaluated by WB analysis, immunofluorescence analysis (IF), and RT-PCR. WB analysis was performed in triplicate, using $\beta$ actin as an internal control. Treatment of $\mathrm{H} 69$ and $\mathrm{H} 1688$ cells with $300 \mathrm{nM}$ and $1000 \mathrm{nM}$ of TSA, respectively, lead to a significant increase in the level of acetylated histones $\mathrm{H} 3$ and $\mathrm{H} 4$, with induction of the Notch1 receptor, compared to control cells. IF staining was performed in triplicate, using Alexa Fluor 488 as a green-emitting signaling molecule for the Notch1-specific antibody, while the signal of the Notch1 intracellular domain (NICD) was detected using Alexa Fluor 568 as a red-emitting signal. All nuclei were counterstained with 4',6-diamidino-2-phenylindole (DAPI, blue). Original magnification, $\times 100$. TSA-treated cells showed expression of Notch1 in cell cytosol, and the expression of NICD in cell nuclei. RT-PCR showed an increased expression level of Notch1 mRNA in TSA-treated cells compared to the control cells. The expression of GAPDH mRNA was used as an internal control. The experiment was performed in triplicate. (b) Representative H\&E and Alcian blue staining photos from subcutaneous tumors arising 28 days after xenotransplantation of $2 \times 10^{6} \mathrm{H} 1688$ cells treated with TSA, and from the control group. The first row represents H\&E staining photos, and shows the glandular cell arrangements (black arrows) in tumors originating from TSA-treated cells compared to the control group. Original magnification $\times 400$ (scale bar $20 \mu \mathrm{m}$ ). The second row represents serial sections stained with Alcian blue. Note the positive reaction in TSA-treated cells at the luminal surfaces of the glandular cell arrangements (black arrows). Original magnification x400 (scale bar $20 \mu \mathrm{m}$ ). (c) Representative IHC staining photos for subcutaneous tumors arising from TSA-treated SCLC cells compared to the control group. The first row represents IHC staining for the Notch1 receptor. Note the positive Notch1 cytosolic reaction (brown) in TSA-treated cells compared to the control cells. Original magnification: $x 40$ (scale bar $200 \mu$ m). The second row represents serial sections IHC stained for syneptophysin (SYP), a neuroendocrine (NE) differentiation marker. Note the decreased cytoplasmic reaction (brown) for SYP in TSA-treated cells compared to the control cells. Original magnification $\times 40$ (scale bar $200 \mu$ m). 
Table 3 Change in Histone H3 Acetylation at the promoter region of the Notch1 gene by TSA Treatment in human SCLC cell Lines

\begin{tabular}{llrr}
\hline \multirow{2}{*}{$\begin{array}{l}\text { Cell } \\
\text { line }\end{array}$} & Condition & \multicolumn{2}{c}{ Acetylated histone H3 } \\
\cline { 3 - 4 } & & Primer 1 & Primer 2 \\
\hline H69 & Control & $2.25 \pm 0.81$ & $2.73 \pm 0.38$ \\
& TSA (300 nM) & $2.72 \pm 0.60$ & $3.15 \pm 0.24$ \\
H1688 & Control & $2.098 \pm 0.63$ & $1.91 \pm 0.45$ \\
& TSA (1000 nM) & $6.72 \pm 3.57$ & $4.91 \pm 2.30$ \\
\hline
\end{tabular}

Cells were treated with control (DMSO) or 300-1000 nM TSA for $24 \mathrm{~h}$. The fold enrichment of the histone acetylation level is expressed. The average from three independent experiments \pm s.d. A ratio of enrichment (TSA treatment/non-treatment) of $>2.0$ was considered to be an increase in the histone acetylation, and these are underlined.

Our results showed that the two components of cSCLC are different from each other, but similar to their respective "pure" counterparts, and that Notch1 is only expressed in the NSCLC component. To clarify the importance of histone acetylation in Notch1 silencing in SCLC, we evaluated the expression of acetylated histone $\mathrm{H} 3$ in lung cancer cells. By WB analysis, there was significant difference between both SCLC and NSCLC cells. Using ChIP assay, we further measured the level of acetylated histone $\mathrm{H} 3$ around the promoter region of the Notch1 gene in lung cancer cells, and were able to identify high levels of acetylation in cells expressing the Notch1 protein in both SCLC and NSCLC cell lines. This is the first report that confirmed the role of histone acetylation in Notch1 expression in lung cancer cells.

In addition, to confirm this finding, we measured the level of acetylated histone $\mathrm{H} 3$ around the Notch 1 promoter in two SCLC cells treated with the HDACi, TSA. One of the treated SCLC cells (H1688) showed a significant increase in the level of acetylated histone $\mathrm{H} 3$ at the Notch1 promoter after TSA treatment, possibly due to the high dose of TSA used for treatment, indicating that an increased acetylated histone $\mathrm{H} 3$ level at the Notch1 promoter, alongside other possible mechanisms, ${ }^{13,14}$ could be attributed to the activation of Notch1 signaling in SCLC after HDACi treatment.

Such differences in Notch1/acetylated histone H3 expression could possibly signify that epigenetic modifications can occur in a 'pure' lung carcinoma cell type (whether SCLC or NSCLC), leading to the concomitant appearance of other components within the same tumor. Our findings were in accordance with previous studies ${ }^{3,26}$ that reported similarities between the two components of cSCLC, indicating a monoclonal origin of the two components that underwent divergent differentiation. In these reports, the authors investigated cases of combined small-cell/large-cell neuroendocrine carcinomas, and compared their genetic alterations to their respective pure forms. ${ }^{3}$ There were similar frequencies of allelic imbalance in each component of combined neoplasms $v s$ their respective pure forms, ${ }^{3}$ and in addition the two components showed a higher degree of genetic concordance. $^{3,31}$

In addition, we aimed to examine the morphology of TSA-treated SCLC cells, and whether the morphology of cells changes after the enrichment of acetylated histones and Notch1 restoration, leading to the concurrent appearance of the NSCLC component. Xenotransplanted H1688 cells treated with TSA retained their cytoplasmic Notch1 expression and showed multiple foci of glandular cell arrangement, with positive Alcian blue staining and reduced staining for neuroendocrine markers. These findings were similar to our previous observations regarding subcutaneous tumors arising from H1688 cells transfected with the Notch1 NICD plasmid. ${ }^{6}$ These observations indicate the appearance of "epithelial-like cell features" in SCLC after the increase in the histone acetylation level of cells that underwent HDACi treatment, despite no significant changes in cellular morphology being observed. This indicates the important role of epigenetic modification, especially histone acetylation, in the histogenesis of cSCLC. In accordance with this, Meder et al. $(2015)^{32}$ postulated that cSCLC could have an NSCLCdependent secondary tumor origin through inactivating Notch mutations and bi-allelic loss of TP53 and RB1. Moreover, increased ASCL1 expression in NSCLC lead to the concomitant appearance of a SCLC component. ${ }^{33}$ Whether cSCLC arises from SCLC that acquires Notch expression, or from NSCLC that loses Notch expression, is a subject for further investigation. In addition, we showed that TSA treatment of SCLC cells resulted in increased cell adhesion, decreased expression of epithelial mesenchymal transition markers, decreased cell proliferation, and activated cell apoptosis. These effects may be related to TSA induction of Notch1 expression, as we previously showed, ${ }^{6,7}$ even in H1688 cells, in which Notch1 plasmid transfection could not affect cell adhesion or epithelial mesenchymal transition markers, ${ }^{7}$ which signifies the potency of TSA treatment on SCLC.

\section{SUMMARY AND CONCLUSION}

Although cSCLC represents a less common variant of SCLC, its high mortality rate and failure of modality treatment necessitates a better understanding of the origin of these tumors. The present study attempted to reveal a possible role for histone acetylation in the origin of cSCLC. We have shown a significant correlation between the level of acetylation of histone $\mathrm{H} 3$ surrounding the promoter region of Notch1, and its expression in human lung cancer cell lines. The results indicated that histone deacetylation plays an important role in Notch1 silencing in SCLC, and that restoration of Notch1 expression in SCLC cells could lead to the concurrent appearance of epithelial-like areas within the SCLC. These findings may indicate that the two components of cSCLC have a common cell origin, and that 
several epigenetic mechanisms, especially histone acetylation, could occur in one of these components and lead to the appearance of the others. However, further studies aimed at examining other possible epigenetic mechanisms are necessary to uncover the pathogenesis of cSCLC.

Supplementary Information accompanies the paper on the Laboratory

Investigation website (http://www.laboratoryinvestigation.org)

\section{ACKNOWLEDGMENTS}

We thank Mrs Motoko Kagayama and Mrs Takako Maeda for their skillful technical assistance. This study was partially supported by a Grant-in-Aid for Scientific Research (C; No.25460439) from the Ministry of Education, Culture, Sports, Science, and Technology of Japan.

\section{DISCLOSURE/CONFLICT OF INTEREST}

The authors declare no conflict of interest.

1. Travis WD, Brambilla E, Burke AP, et al. WHO Classification of Tumours of the Lung, Pleura, Thymus and Heart World Health Organization Classification of Tumours. IARC Press: Lyon, France, 2015, pp 10-20.

2. Jemal A, Bray F, Center MM, et al. Global cancer statistics. CA Cancer J Clin 2011:61:69-90.

3. Wagner PL, Kitabayashi N, Chen YT, et al. Combined small cell lung carcinomas: genotypic and immuophenotypic analysis of the separate morphologic components. Am J Clin Pathol 2009;131:376-382.

4. Li Y, Burns JA, Cheney CA, et al. Distinct expression profiles of Notch-1 protein in human solid tumors: Implications for development of targeted therapeutic monoclonal antibodies. Biologics 2010:4:163-171.

5. Kunnimalaiyaan $\mathrm{M}$, Chen $\mathrm{H}$. Tumor suppressor role of Notch1 signaling in neuroendocrine tumors. Oncologist 2007;12:535-542.

6. Wael $\mathrm{H}$, Yoshida $\mathrm{R}$, Kudoh $\mathrm{S}$, et al. Notch1 signaling controls cell proliferation, apoptosis and differentiation in lung carcinoma. Lung Cancer 2014:85:131-140.

7. Hassan WA, Yoshida R, Kudoh $S$, et al. Notch 1 controls cell invasion and metastasis in small cell lung carcinoma cell lines. Lung Cancer 2014;86: 304-310.

8. George J, Lim JS, Jang SJ, et al. Comprehensive genomic profiles of small cell lung cancer. Nature 2015;524:47-53.

9. Barlési F, Giaccone G, Gallegos-Ruiz $\mathrm{Ml}$, et al. Global histone modifications predict prognosis of resected non small-cell lung cancer. J Clin Oncol 2007;25:4358-4364.

10. Mateen S, Raina K, Jain AK, et al. Epigenetic modifications and p21cyclin B1 nexus in anticancer effect of histone deacetylase inhibitors in combination with silibinin on non-small cell lung cancer cells. Epigenetics 2012;7:1161-1172.

11. Roche J, Nasarre P, Gemmill R, et al. Global decrease of histone H3K27 acetylation in ZEB1-induced epithelial to mesenchymal transition in lung cancer cells. Cancers 2013;5:334-356.

12. Li F, Ye B, Hong L, et al. Epigenetic modifications of histone h4 in lung neuroendocrine tumors. Appl Immunohistochem Mol Morphol 2011:19:389-394.

13. Platta CS, Greenblatt DY, Kunnimalaiyaan M, et al. The HDAC inhibitor trichostatin A inhibits growth of small cell lung cancer cells. J Surg Res 2007;142:219-226.

14. Platta CS, Greenblatt $D$, Kunnimalaiyaan $M$, et al. Valproic acid induces Notch1 signaling in small cell lung cancer cells. J Surg Res 2008;148:31-37.
15. Greenblatt DY, Vaccaro AM, Jaskula-Sztul R, et al. Valproic acid activates notch-1 signaling and regulates the neuroendocrine phenotype in carcinoid cancer cells. Oncologist 2007;12:942-951.

16. Daly K, Shirazi-Beechey SP. Microarray analysis of butyrate regulated genes in colonic epithelial cells. DNA Cell Biol 2006;25:49-62.

17. Yoshida $R$, Nagata $M$, Nakayama $H$, et al. The pathological significance of Notch1 in oral squamous cell carcinoma. Lab Invest 2013;93: 1068-1081.

18. Ellisen LW, Bird J, West DC, et al. TAN-1, the human homolog of the Drosophila notch gene, is broken by chromosomal translocations in T lymphoblastic neoplasms. Cell 1991;66:649-661.

19. Takebayashi S, Lei I, Ryba T, et al. Murine esBAF chromatin remodeling complex subunits BAF250a and Brg1 are necessary to maintain and reprogram pluripotency-specific replication timing of select replication domains. Epigenetics Chromatin 2013;6:42.

20. Tani M, Ito J, Nishioka $M$, et al. Correlation between histone acetylation and expression of the MYO18B gene in human lung cancer cells. Genes Chromosomes Cancer 2004;40:146-151.

21. El-Awady RA, Hersi $\mathrm{F}$, AlTunaiji $\mathrm{H}$, et al. Epigenetics and miRNA as predictive markers and targets for lung cancer chemotherapy. Cancer Biol Ther 2015;16:1056-1070.

22. Hajji N, Wallenborg $K$, Vlachos $P$, et al. Combinatorial action of the HDAC inhibitor trichostatin A and etoposide induces caspasemediated AIF-dependent apoptotic cell death in non-small cell lung carcinoma cells. Oncogene 2008;27:3134-3144.

23. Byers LA, Wang J, Nilsson MB, et al. Proteomic profiling identifies dysregulated pathways in small cell lung cancer and novel therapeutic targets including PARP1. Cancer Discov 2012;2:798-811.

24. Sequist LV, Waltman BA, Dias-Santagata D, et al. Genotypic and histological evolution of lung cancers acquiring resistance to EGFR inhibitors. Sci Transl Med 2011;3:75ra26.

25. Brambilla E, Moro D, Gazzeri S, et al. Cytotoxic chemotherapy induces cell differentiation in small-cell lung carcinoma. J Clin Oncol 1991;9: 50-61.

26. Fujino K, Motooka Y, Hassan WA, et al. INSM1 is a crucial regulator of neuroendocrine differentiation in lung cancer. Am J Pathol 2015;185: 3164-3177.

27. Mirski SE, Gerlach JH, Cole SP. Multidrug resistance in a human small cell lung cancer cell line selected in adriamycin. Cancer Res 1987;47: 2594-2598.

28. Glasspool RM, Teodoridis JM, Brown R. Epigenetics as a mechanism driving polygenic clinical drug resistance. BJ Cancer 2006;94: 1087-1092.

29. El-Khoury V, Breuzard G, Fourré $\mathrm{N}$, et al. The histone deacetylase inhibitor trichostatin A downregulates human MDR1 (ABCB1) gene expression by a transcription-dependent mechanism in a drugresistant small cell lung carcinoma cell line model. Br J Cancer 2007;97: 562-573.

30. Ivanov $M$, Kacevska $M$, Ingelman-Sundberg $M$. Epigenomics and interindividual differences in drug response. Clin Pharmacol Ther 2012;92:727-736.

31. Ullmann R, Petzmann S, Sharma A et al. Chromosomal aberrations in a series of large-cell neuroendocrine carcinomas: unexpected divergence from small-cell carcinoma of the lung. Hum Pathol 2001;32: 1059-1063.

32. Meder L, König K, Ozretić L, et al. NOTCH, ASCL1, p53 and RB alterations define an alternative pathway driving neuroendocrine and small cell lung carcinomas. Int J Cancer 2016;138:927-938.

33. Ito $\mathrm{T}$, Kudoh $\mathrm{S}$, Ichimura $\mathrm{T}$, et al. Small cell lung cancer, an epithelial to mesenchymal transition (EMT)-like cancer: Significance of inactive Notch signaling and expression of Achaete-scute complex homologue 1. Hum Cell 2017;30:1-10. 